\title{
Rúbrica analítica para evaluar la inteligencia emocional a través de un cuestionario diagnóstico
}

\author{
Vicente Román-Acosta \\ vicente@integrandoequipos.com \\ Integrando Equipos S.C., México \\ Paula Flora Aniceto-Vargas \\ paulafloraa@gmail.com \\ Instituto Politécnico Nacional, México \\ Rebeca Román-Julián \\ rroman@unach.mx \\ Universidad Autónoma de Chiapas, México
}

\section{RESUMEN}

Desde la socioformación, las rúbricas permiten evaluar el desempeño en la realización de procesos o evidencias y tiene como propósito aportar conocimiento sobre las competencias socioemocionales ante el creciente interés en el diseño de instrumentos que posibiliten la identificación y evaluación de las competencias profesionales, personales o sistémicas. Se realizó un estudio instrumental para la construcción y validación de una rúbrica analítica para evaluar la competencia socioemocional en adultos, específicamente respecto a la inteligencia emocional, mediante la identificación de factores que permitieron proponer un instrumento fiable y válido para su aplicación. El procedimiento consistió en el diseño de la rúbrica, una revisión de expertos, la validación de contenido mediante la técnica de juicio de expertos y el método de la $\mathrm{V}$ de Aiken y el análisis de confiabilidad con Alfa de Cronbach, producto de la aplicación a un grupo piloto. Los resultados encontrados llevaron a plantear la necesidad de perfilar mejor las variables medidas por los cuestionarios diagnósticos y a cuestionar si los actuales instrumentos de medida de la inteligencia emocional (IE) realmente reúnen evidencias de validez científica.

Palabras clave: competencia; cuestionario; evaluación; inteligencia emocional; juicio de expertos 


\title{
Analytical rubric to evaluate the emotional intelligence through a diagnostic questionnaire
}

\begin{abstract}
The purpose of the research presented is to provide greater knowledge about socioemotional competencies, given the growing interest in the design of instruments that enable the identification and evaluation of professional, personal or systemic competencies. An instrumental study was carried out for the construction and validation of an analytical rubric to evaluate Socioemotional Competence in adults (CSE), specifically with regard to emotional intelligence, by identifying factors that allow proposing a reliable and valid instrument for its application. The procedure consisted of the design of the rubric, an expert review, the content validation using the expert judgment technique and the Aiken's V method, and the reliability analysis with Cronbach's Alpha, product of the application to a pilot group. The results found lead to the need to better define the variables measured by the diagnostic questionnaires and to question whether the current instruments for measuring emotional intelligence (EI) really gather evidence of construct validity.
\end{abstract}

Keywords: competence; evaluation; emotional intelligence; expert judgment; questionnaire

Artículo recibido: 05 octubre. 2021 Aceptado para publicación: 02 noviembre 2021 Correspondencia: vicente@integrandoequipos.com Conflictos de Interés: Ninguna que declarar 


\section{INTRODUCCIÓN}

Producto del interés en el estudio de la inteligencia emocional y de identificarla dentro del ámbito de las competencias emocionales definidas por Bisquerra (2003, p. 328), como "un conjunto de conocimientos, capacidades, habilidades y actitudes necesarias para comprender, expresar y regular apropiadamente los fenómenos emocionales”, la problemática que da origen a esta investigación es la inexistencia de un consenso acerca de las variables que la conforman, lo cual invita a efectuar un análisis de las dimensiones que los principales autores en el tema han propuesto, a fin de integrar aquellas que resulten comunes en los diferentes planteamientos teóricos.

Como lo hacen notar López-Goñi y Goñi (2012) las competencias socioemocionales son conocimientos, habilidades y actitudes sociales y emocionales, puestos en práctica en la vida real de las personas, en este sentido, su desarrollo en la formación profesional y personal se sitúa como una necesidad que se deriva, por una parte, de las nuevas concepciones filosóficas que critican el excesivo énfasis que se ha puesto en la razón instrumental como forma de comprensión de la realidad y, por otra, de una visión que contempla al profesional de empresas con personal a cargo como alguien que debe desarrollar las competencias mencionadas como un eje en torno al que gire su formación. La unión de estas dos visiones permite justificar la conveniencia de introducir las competencias emocionales en los currículos de formación de los profesionales.

De acuerdo con Durlak, Weissberg, Dymnicki, Taylor y Schellinger (2011) desde finales del siglo XX los profesionales en las organizaciones han ido tomando conciencia de la importancia del desarrollo integral de ellos mismos y las personas que dirigen, incluyendo las competencias académicas y las competencias socioemocionales, entre las que destaca la Inteligencia Emocional (IE) como la capacidad para percibir, utilizar, expresar y regular las emociones, basándose en el paradigma cognitivo de la educación, atendiendo exclusivamente a las habilidades de procesamiento de la información emocional (Mayer y Salovey, 1997).

En ese sentido Zych, Beltrán-Catalán, Ortega-Ruiz y Llorent (2017) proponen un conjunto de competencias que incluyen el autoconocimiento, el autocontrol, la conciencia social y prosocialidad, y la toma de decisiones responsable, factores incorporados dentro de los ítems y dominios del constructo teórico de la investigación.

Con base en los antecedentes descritos, el estudio empírico realizado presenta la 
construcción y validación de una rúbrica analítica para medir las Competencias Socioemocionales en adultos (CSE), en relación con la inteligencia emocional. En función de este criterio se definió conformado por las dimensiones: 1) Percepción emocional propia, 2) Percepción emocional de otros y 3) Adaptación y enfoque emocional, siguiendo un proceso que comprendió las siguientes etapas:

a) Definición conceptual

b) Elaboración de los ítems

c) Análisis de la validez de contenido

d) Evaluación por juicio de expertos

e) Análisis y selección de reactivos

f) Prueba piloto

Se elige estudiar de manera específica la inteligencia emocional, ya que se considera una de las competencias socioemocionales más abordadas por investigadores, docentes y colaboradores en las organizaciones a nivel latinoamérica, generando diferentes posiciones teóricas. A partir de la primera publicación de Gardner (2011) se reformuló el concepto de inteligencia, en donde se establece que los seres humanos poseen siete tipos de inteligencia, cada una independiente de las otras, planteamiento que dió inicio a todo un cambio en la perspectiva acerca de lo que se entendía hasta el momento por inteligencia.

Desde la perspectiva de García-Fernández y Giménez-Mas (2010, p. 45) la inteligencia emocional se define como "la capacidad para reconocer nuestros propios sentimientos y los de los demás, para motivarse y gestionar la emocionalidad en nosotros mismos y en las relaciones interpersonales", lo que enfatiza la necesidad de la conciencia y el entrenamiento para desarrollar las competencias socioemocionales en función del desarrollo personal y profesional de los colaboradores en las organizaciones.

La importancia de la necesidad planteada en esta investigación, radica en generar una propuesta para evaluar la competencia socioemocional específica de inteligencia emocional, ya que se asume que todas las personas presentan diferencias individuales en ella y por tanto tienen diferentes capacidades para atender sus emociones. Para ello, tanto en la literatura como en el ámbito corporativo existen diferentes cuestionarios, evaluaciones o pruebas, mismos que pretenden evaluar los diversos componentes de la inteligencia emocional como competencia esencial de los colaboradores, encontrándose 
por un lado los mixtos, enfocados en aspectos de la personalidad en función de la inteligencia emocional y los de habilidades con base en la percepción y reacción con la inteligencia emocional.

El desarrollo de este estudio surge del interés por crear una herramienta cuantitativa confiable para el entorno organizacional, mediante el diseño y validación de un instrumento para medir la competencia socioemocional de inteligencia emocional, por ello, teniendo como propósito dar certidumbre a los resultados del cuestionario y presentar un acercamiento a los autores que han contribuido tanto teórica como empíricamente a establecer la inteligencia emocional desde una visión rigurosa, se identificó un vacío o dificultad para encontrar instrumentos con validez científica para evaluar la competencia estudiada y que a su vez le permitan al usuario:

1) Responder un cuestionario diagnóstico en función de la inteligencia emocional, mismo que le permita saber cuáles son sus áreas de mejora.

2) Observar y generar un plan de acción que le ayude a mitigar las áreas de oportunidad en función de la competencia socioemocional de inteligencia emocional.

3) Aplicar soluciones que le permitan dirigir de forma eficiente al personal a cargo.

\section{METODOLOGÍA}

\section{Tipo de Estudio}

A juicio de Montero y León (2005), el estudio fue realizado como un producto instrumental por orientarse al desarrollo de una prueba, incluyendo tanto el diseño o adaptación, como el estudio de las propiedades psicométricas del mismo, en este caso respecto a una rúbrica analítica para evaluar la competencia socioemocional de inteligencia emocional.

\section{Instrumento}

El propósito primario del instrumento ha sido el de evaluar la inteligencia emocional como competencia socioemocional mediante una escala tipo Likert, dirigido a personas de sexo indistinto, con condiciones económicas estables, situadas en una región latinoamericana, económicamente activas, con personal a cargo en cualquier área de gestión a través de los diferentes puestos organizacionales, que deseen identificar sus áreas de mejora mediante una prueba con validez científica.

La rúbrica analítica evalúa la inteligencia emocional como competencia socio emocional y fué conformada por tres dimensiones independientes, que son: 1) Percepción emocional 
propia, 2) Percepción emocional de otros y 3) Adaptación y enfoque emocional, a través de quince ítems o niveles de desempeño con cuatro niveles de respuesta con base en una taxonomía socioformativa que los clasifica como: Receptivo, Resolutivo, Autónomo y Estratégico.

La información recabada para fundamentar teóricamente el diseño del instrumento procedió de bases de datos pertinentes (Sciendirect, Scielo, Latindex, Google Académico), seleccionando principalmente artículos de revistas indexadas y libros en español e inglés que incluyeran los términos validez de contenido, juez y juicio de expertos. La revisión se hizo por parte del juicio de tres expertos, la validez de contenido por veinte jueces expertos y la evaluación de la confiabilidad con la participación de catorce personas en un grupo piloto con base en el cual se determinó un grado muy bueno de comprensión de instrucciones, redacción y pertinencia de los ítems y se realizó un análisis de consistencia interna, el cual reveló un valor aceptable con un alfa de Cronbach igual a 0.8249 .

\section{Participantes}

Para la revisión del instrumento se contó con tres expertos que se eligieron por su competencia y tuvieron la disposición de ayudar en el proceso para brindar su punto de vista en función del instrumento planeado. Sus datos sociodemográficos se describen en la Tabla 1.

Tabla 1. Datos sociodemográficos de expertos

\begin{tabular}{ll}
\hline Sexo & $\begin{array}{l}\mathbf{1} \text { hombre } \\
\mathbf{2} \text { mujeres }\end{array}$ \\
\hline Promedio de edad: & 43 años \\
\hline Estado civil: & 2 solteros, 1 casado \\
\hline Promedio de años de estudio: & 21 años \\
\hline Condiciones económicas: & Estables \\
\hline Zona de residencia: & $\begin{array}{l}\text { Monterrey, México; } \\
\text { San Luis Potosí, México y } \\
\text { Santiago de Chile, Chile }\end{array}$ \\
\hline Situación laboral: & $\begin{array}{l}\text { Docentes o consultores especializados en } \\
\text { competencias independientes }\end{array}$ \\
\hline
\end{tabular}

Fuente: Elaboración propia, adaptada de Hernández-Mosqueda, Tobón-Tobón y Guerrero-Rosas (2016). 
Los veinte expertos que participaron en el proceso de validación por juicio de expertos cuentan con los atributos sociodemodráficos que se presentan en la Tabla 2. Los jueces se eligieron por su competencia en la planeación y experiencia en investigaciones empíricas de diferentes regiones de Latinoamérica y brindaron el apoyo para realizar su evaluación en función de la propuesta.

Tabla 2. Datos sociodemográficos de jueces expertos

\begin{tabular}{ll}
\hline Sexo & $\begin{array}{l}\mathbf{6} \text { hombres } \\
\mathbf{1 4} \text { mujeres }\end{array}$ \\
\hline Promedio de edad: & 48 años \\
Estado civil: & 15 casados, \\
Promedio de años de & 23 años \\
estudio: & Estables \\
Condiciones & \\
económicas: & México, Colombia, Perú, San Salvador, Ecuador, \\
Zona de residencia: & Bolivia, Argentina y Guatemala. \\
& Docentes, \\
Situación laboral: & emprendedores, consultores, especialistas en docencia \\
& e investigación.
\end{tabular}

Fuente: Elaboración propia, adaptada de Ibarra-Piza, Segredo-Santamaria, JuárezHernández \& Tobón-Tobón (2018).

Para evaluar la confiabilidad del instrumento participaron seis hombres y ocho mujeres, con condiciones económicas estables, todos con personal a cargo de empresas diversas en México, Colombia y Perú, como se especifica en la Tabla 3. El grupo piloto tuvo la disponibilidad de participar en el estudio y aplicación práctica en el proceso de mediación y posteriormente en el auto diagnóstico en función del constructo teórico en cuestión, producto de lo cual aportaron áreas de mejora. 
Tabla 3. Datos sociodemográficos del grupo piloto

\begin{tabular}{ll}
\hline Sexo & $\begin{array}{l}\text { 6 hombres } \\
\mathbf{8} \text { mujeres }\end{array}$ \\
\hline Promedio de edad: & $\begin{array}{l}35 \text { años } \\
9 \text { casados, } \\
5 \text { solteros }\end{array}$ \\
Estado civil: & $\begin{array}{l}18 \text { años } \\
\text { Promedio de años de } \\
\text { estudio: }\end{array}$ \\
$\begin{array}{l}\text { Condiciones } \\
\text { económicas: }\end{array}$ & Estables \\
Zona de residencia: & México, Colombia, Perú \\
& \\
Situación laboral: & $\begin{array}{l}\text { Contratados por organizaciones y con personal funcional a } \\
\text { cargo }\end{array}$ \\
\hline
\end{tabular}

Fuente: Elaboración propia, adaptada de Hernández-Mosqueda, Tobón-Tobón y

Guerrero-Rosas (2016).

\section{Procedimiento}

Con la finalidad de garantizar la validez de contenido y la confiabilidad del instrumento se siguieron los pasos que se describen a continuación.

\section{Diseño de la rúbrica analitica socioformativa}

Una vez definido el constructo teórico de interés relativo a medir la competencia socioemocional de inteligencia emocional, se realizó el diseño de una rúbrica socioformativa que quedó conformada por tres dimensiones independientes: 1) Percepción emocional propia, 2) Percepción emocional de otros y 3) Adaptación y enfoque emocional, a través de 15 ítems con cuatro niveles de respuesta basados en una taxonomía socioformativa que los clasifica como: Receptivo, Resolutivo, Autónomo y Estratégico.

\section{Revisión del instrumento}

La revisión se hizo con la participación de tres expertos en el área de la docencia y consultoría en competencias socioemocionales, con al menos diez años de experiencia cada uno en función del concepto, metodología y aplicación de didáctica con relación a la inteligencia emocional 


\section{Validez de contenido}

La nueva versión de la rúbrica con las mejoras sugeridas en la etapa de revisión se sometió a un juicio de veinte expertos, no en el tema, pero sí en la planeación y diseño de instrumentos cuantitativos, empíricos y de rigor científico que, como lo señalan Muñiz, Suárez-Álvarez, Pedrosa, Fonseca-Pedrero y García-Cueto (2014) es el método para validar juicios. La validez que realizaron respecto a la pertinencia y redacción de cada ítem, se basó en una escala del 1 al 4 en donde 1 significó un nivel Muy bajo y 4 Muy alto, aportando además sus observaciones para mejorarlos.

De acuerdo con la metodología seguida se hicieron las modificaciones necesarias, considerando las sugerencias hechas por los expertos y se realizó una segunda fase del proceso de juicio de experto con los mismos participantes y en ambas etapas se utilizó la $\mathrm{V}$ de Aiken como método centrado en la relevancia ítem-constructo, tomando en cuenta el número de expertos participantes.

\section{Determinación de la Confiabilidad}

Posteriormente a las dos fases del juicio de expertos se invitó a quince personas que corresponde a la población objetivo, para conformar el grupo piloto que permitió evaluar la confiabilidad del instrumento. Se llevó a cabo un proceso de mediación para compartir el constructo teórico, lo que pretende el instrumento y la descripción de los ítems y sus dimensiones, con la finalidad de conocer, por un lado, que las instrucciones y los ítems fueran comprensibles y por otro, el grado respecto a la redacción, pertinencia y satisfacción en función de la muestra a la que se le aplicará la rúbrica.

\section{Análisis estadístico}

Para determinar la validez de contenido se utilizó el método de la V de Aiken en las dos fases que participaron los jueces, obtenida en función de cada uno de ellos respecto a la pertinencia y redacción de cada uno de los ítems pertenecientes a los tres dimensiones del constructo teórico presentado (Aiken, 1985).

En lo que se refiere al análisis de confiabilidad se utilizó el Alfa de Cronbach determinada por medio del software estadístico SPSS y que, de acuerdo con Welch y Comer (1988), permite estimar la fiabilidad de un instrumento de tal forma que si su valor es más cercano a 1 hay mayor consistencia interna de los ítems, lo que significa que miden el constructo que se pretende medir. La pertinencia promedio en función de los quince ítems fue de 0.89 y la redacción promedio fue de 0.8411 respectivamente. 


\section{RESULTADOS}

\section{Diseño de la rúbrica analítica socioformativa}

Después de considerar las sugerencias de mejora recomendadas por los expertos, el juicio de expertos y el grupo piloto, se obtuvo la rúbrica analítica socioformativa que se describe a continuación.

Tabla. 4. Dimensión 1) Percepción emocional propia

Indicadores o criterios

1.Un sentimiento es un estado afectivo del ánimo o disposición emocional hacia una cosa, en función de ello: ¿En qué nivel auto evalúa sus propios sentimientos en términos generales?

\section{Escala taxonómica socioformativa} (Niveles de desempeño)

(1) Nivel receptivo: Con frecuencia identifico mis propios sentimientos.

(2) Nivel resolutivo: Con frecuencia proceso mis propios sentimientos.

(3) Nivel autónomo: Con frecuencia analizo mis propios sentimientos.

(4) Nivel estratégico: Con frecuencia adapto y transformo mis propios sentimientos.
2.Una preocupación es la acción y efecto de preocuparse, es un estado de desasosiego, inquietud o temor producido ante una situación difícil, en función de ello:

¿En qué nivel identifica lo que siente en función de lo que le preocupa en términos generales?
(1) Nivel receptivo: Indaga acerca de lo que le preocupa.

(2) Nivel resolutivo: Comprende lo que le preocupa.

(3) Nivel autónomo: Controla su preocupación.

(4) Nivel estratégico: Transfiere su preocupación a lo que siente

3. Entendiendo estado de ánimo como la disposición en que se encuentra alguien causada por la alegría, la tristeza, el abandono, etc. El humor o tono sentimental, agradable o desagradable, que acompaña a una idea o situación y se mantiene por algún tiempo. Es una forma de estar o permanecer, que expresa matices afectivos y cuya duración es prolongada, de horas o días y en función de ello: ¿En qué nivel auto evalúa su

(1) Nivel receptivo: Identifica su estado de ánimo.

(2) Nivel resolutivo: Comprende lo que le preocupa.

(3) Nivel autónomo: Analiza su estado de ánimo.

(4) Nivel estratégico: Juzga su estado de ánimo. 
4. La empatía se describe como un sentimiento de identificación con algo o alguien, o la capacidad de identificarse con alguien y compartir sus sentimientos, en función de ello: ¿En qué nivel percibe su empatía en términos generales?
(1) Nivel receptivo: Reconoce los sentimientos de otros en usted.

(2) Nivel resolutivo: Procesa los sentimientos de otros en usted.

(3) Nivel autónomo: Regula los sentimientos de otros en usted.

(4) Nivel estratégico: Vincula los sentimientos de otros en usted.

5.La inteligencia interpersonal es definida como la capacidad de comprender a los demás. Afirma que las personas que la han desarrollado son capaces de discernir y responder apropiadamente a los estados de ánimo, temperamentos, motivaciones $\mathrm{y}$ deseos de las demás personas, en función de ello: ¿En qué nivel identifica su inteligencia interpersonal en términos generales?

6.La inteligencia intrapersonal permite formar una imagen precisa de nosotros mismos; permite poder entender nuestras necesidades y características, así como nuestras cualidades y defectos, en función de ello: ¿En qué nivel identifica su inteligencia intrapersonal en términos generales?
(1) Nivel receptivo: Busca su capacidad de comprensión de los demás.

(2) Nivel resolutivo: Comprende su capacidad de comprensión de los demás.

(3) Nivel autónomo: Analiza su capacidad de comprensión de los demás.

(4) Nivel estratégico: Predice su capacidad de comprensión de los demás.

(1) Nivel receptivo: Reconoce su imagen precisa para entender las necesidades propias.

(2) Nivel resolutivo: Comprueba su imagen precisa para entender las necesidades propias.

(3) Nivel autónomo: Autogestiona su imagen precisa para entender las necesidades propias.

(4) Nivel estratégico: Adapta su imagen precisa para entender las necesidades propias

\section{Fuente: Elaboración propia.}

Tabla 5. Dimensión 2) Percepción emocional de otros

\section{Indicadores $\boldsymbol{o}$ criterios}

\section{Escala taxonómica socioformativa}

(Niveles de desempeño)

(1) Nivel receptivo: Identifica los sentimientos de otros en usted.

7.Un sentimiento es un estado afectivo del ánimo o disposición emocional hacia una cosa, en función de ello: ¿En qué nivel percibe los sentimientos de otras personas en términos generales?
(2) Nivel resolutivo: Interpreta los sentimientos de otros en usted.

(3) Nivel autónomo: Coevalúa los sentimientos de otros en usted.

(4) Nivel estratégico: Adapta los sentimientos de otros en usted. 
8. Entendiendo estado de ánimo como la disposición en que se encuentra alguien causada por la alegría, la tristeza, el abandono, etc. El humor o tono sentimental, agradable o desagradable, que acompaña a una idea o situación y se mantiene por algún tiempo. Es una forma de estar o permanecer, que expresa matices afectivos y cuya duración es prolongada, de horas o días y en función de ello: ¿En qué nivel percibe el estado de ánimo de otras personas en términos generales?
(1) Nivel receptivo: Reconoce los sentimientos de otros en usted.

(2) Nivel resolutivo: Procesa los sentimientos de otros en usted.

(3) Nivel autónomo: Regula los sentimientos de otros en usted.

(4) Nivel estratégico: Vincula los sentimientos de otros en usted.

9. Entendiendo estado de ánimo como la disposición en que se encuentra alguien causada por la alegría, la tristeza, el abandono, etc. El humor $\mathrm{o}$ tono sentimental, agradable o desagradable, que acompaña a una idea o situación y se mantiene por algún tiempo. Es una forma de estar o permanecer, que expresa matices afectivos y cuya duración es prolongada, de horas o días y en función de ello: ¿En qué nivel acompaña el estado

(1) Nivel receptivo: Identifica el estado de ánimo de otras personas.

(2) Nivel resolutivo: Comprende el estado de ánimo de otras personas.

(3) Nivel autónomo: Analiza el estado de ánimo de otras personas.

(4) Nivel estratégico: Juzga el estado de ánimo de otras personas. de ánimo de otras personas en términos generales?

10. Entendiendo estado de ánimo como la disposición en que se encuentra alguien causada por la alegría, la tristeza, el abandono, etc. El humor o tono sentimental, agradable o desagradable, que acompaña a una idea o situación y se mantiene por algún tiempo. Es una forma de estar o permanecer, que expresa matices afectivos y cuya duración es prolongada, de horas o días y en función de ello: ¿En qué nivel le impacta el estado de ánimo de otras personas en términos generales?
(1) Nivel receptivo: Define mi estado de ánimo.

(2) Nivel resolutivo: Motiva mi estado de ánimo.

(3) Nivel autónomo: Infiere en mi estado de ánimo.

(4) Nivel estratégico: Transforma mi estado de ánimo. 
11. Entendiendo estado de ánimo como la disposición en que se encuentra alguien causada por la alegría, la tristeza, el abandono, etc. El humor o tono sentimental, agradable o desagradable, que acompaña a una idea o situación y se mantiene por algún tiempo. Es una forma de estar o permanecer, que expresa matices afectivos y cuya duración es prolongada, de horas o días y en función de ello: ¿En qué nivel influye a nivel personal en el estado de ánimo de otras personas en términos generales?
(1) Nivel receptivo: Define el estado de ánimo de otras personas.

(2) Nivel resolutivo: Motiva el estado de ánimo de otras personas.

(3) Nivel autónomo: Integra el estado de ánimo de otras personas.

(4) Nivel estratégico: Transforma el estado de ánimo de otras personas.

Fuente: Elaboración propia.

Tabla 6. Dimensión 3) Adaptación y enfoque emocional

\section{Indicadores $\boldsymbol{o}$ criterios}

12. Entendiendo estado de ánimo como la disposición en que se encuentra alguien causada por la alegría, la tristeza, el abandono, etc. El humor o tono sentimental, agradable o desagradable, que acompaña a una idea o situación y se mantiene por algún tiempo. Es una forma de estar o permanecer, que expresa matices afectivos y cuya duración es prolongada, de horas o días y en función de ello: ¿En qué nivel se adapta al estado de ánimo de otras personas en términos generales?
Escala taxonómica socioformativa (Niveles de desempeño)

(1) Nivel receptivo: Identifico el nivel de adaptación personal en función del estado de ánimo de otras personas.

(2) Nivel resolutivo: Implemento el nivel de adaptación personal en función del estado de ánimo de otras personas.

(3) Nivel autónomo: Regulo el nivel de adaptación personal en función del estado de ánimo de otras personas.

(4) Nivel estratégico: Personalizo el nivel de adaptación personal en función del estado de ánimo de otras personas.

13. Entendiendo estado de ánimo como el humor o tono sentimental, agradable o desagradable, que acompaña a una idea o situación y se mantiene por algún tiempo. Es un estado, una forma de estar o permanecer, que expresa matices afectivos y cuya duración es prolongada, de horas o días En función de ello: ¿En qué nivel percibe su propio estado de ánimo en términos generales?

(1) Nivel receptivo: Identifico mi estado de ánimo.

(2) Nivel resolutivo: Controlo mi estado de ánimo.

(3) Nivel autónomo: Reflexiono mi estado de ánimo.

(4) Nivel estratégico: Adapto mi estado de ánimo. 
14. Entendiendo estado de ánimo como la disposición en que se encuentra alguien causada por la alegría, la tristeza, el abandono, etc. El humor o tono sentimental, agradable o desagradable, que acompaña a una idea o situación y se mantiene por algún tiempo. Es una forma de estar o permanecer, que expresa matices afectivos y cuya duración es prolongada, de horas o días y en función de ello: ¿En qué nivel adapta su estado emocional ante situaciones sorpresivas en términos generales?
(1) Nivel receptivo: Defino el grado de adaptabilidad a situaciones sorpresivas.

(2) Nivel resolutivo: Interpreto el grado de adaptabilidad a situaciones sorpresivas.

(3) Nivel autónomo: Analizo el grado de adaptabilidad a situaciones sorpresivas.

(4) Nivel estratégico: Transformo el grado de adaptabilidad a situaciones sorpresivas.

15. Entendiendo estado de ánimo como la disposición en que se encuentra alguien causada por la alegría, la tristeza, el abandono, etc. El humor o tono sentimental, agradable o desagradable, que acompaña a una idea o situación y se mantiene por algún tiempo. Es una forma de estar o permanecer, que expresa matices afectivos y cuya duración es prolongada, de horas o días y en función de ello: ¿En qué nivel le ayuda saber su

(1) Nivel receptivo: Registro mi estado de ánimo.

(2) Nivel resolutivo: Interpreto mi estado de ánimo.

(3) Nivel autónomo: Autoevalúo mi estado de ánimo.

(4) Nivel estratégico: Adapto mi estado de ánimo. estado emocional ante un problema en términos generales?

\section{Fuente: Elaboración propia.}

\section{Revisión del instrumento}

En función de lo planteado en el apartado anterior, donde se describen a detalle las dimensiones, los criterios y los niveles de desempeño definitivos de la rúbrica analítica, se consideró la revisión de tres expertos en el tema de la inteligencia emocional. El análisis procedente deriva de las coincidencias que se encontraron en función a la pertinencia de la rúbrica y se tuvieron sugerencias de mejora que fueron atendidas. 
Tabla 7. Sugerencias de los expertos para mejorar el instrumento

\begin{tabular}{|c|c|c|c|}
\hline Dimensión & Pertinencia & Redacción & $\begin{array}{l}\text { Sugerencias de mejora en función al } \\
\text { instrumento }\end{array}$ \\
\hline $\begin{array}{l}\text { Percepción } \\
\text { emocional } \\
\text { propia }\end{array}$ & & $X$ & $\begin{array}{l}\text { Distinguir los niveles de desempeño en } \\
\text { función a la auto evaluación de los } \\
\text { sentimientos propios, distinguir entre estado } \\
\text { de ánimo y sentimiento, Sugiero el orden de: } \\
\text { Identifica, Analiza, Procesa y transforma, } \\
\text { sugiere hacer la distinción entre evaluar e } \\
\text { identificar, los verbos de acción son poco } \\
\text { entendibles. }\end{array}$ \\
\hline $\begin{array}{l}\text { Percepción } \\
\text { emocional de } \\
\text { otros }\end{array}$ & & $X$ & $\begin{array}{l}\text { Cambiar "otros" por otras personas", La } \\
\text { palabra "enuncia" me resulta algo lejana } \\
\text { para vincularla a sentimientos. Se propone } \\
\text { "revela, muestra" }\end{array}$ \\
\hline $\begin{array}{l}\text { Adaptación y } \\
\text { enfoque } \\
\text { emocional }\end{array}$ & & $X$ & $\begin{array}{l}\text { Propone dar contexto de los idicadores y } \\
\text { criterios para que el ususario distinga los } \\
\text { conceptos para responder, distinguir entre } \\
\text { adaptabilidad y adaptación, clarificar si } \\
\text { determinar se refiere a dictaminar o asignar } \\
\text { una emoción específica. }\end{array}$ \\
\hline
\end{tabular}

Fuente: Elaboración propia.

\section{Validez de contenido}

En el análisis de validez de contenido mediante el coeficiente denominado V de Aiken, se cuantificó la relevancia de cada ítem relacionado a la pertinencia y a la redacción respectivamente, evaluado por veinte jueces oscilando desde .7333 como valor inferior hasta .9833 , siendo el valor de uno indicativo de un perfecto acuerdo entre los jueces respecto a la mayor puntuación de validez de los contenidos evaluados, que en el caso de esta investigación generó un valor de 0.89 .

\section{Confiabilidad y análisis estadístico}

Producto de un análisis con el método de Alfa de Cronbach se determinó que el instrumento posee consistencia interna y, por lo tanto, es confiable, con un valor de 0.824 como se aprecia en la tabla 8 , que se considera de bueno a excelente, ya que los ítems se ubicaron en una valoración alta y la concordancia interna entre uno y otro ítem fue satisfactoria. Si bien en términos generales los ítems evaluados dieron como resultado un 
Alfa de Cronbach de 0.824, se destacan los ítems 1, 3, 4, 5, 6, 11, 14 y 15 que en función a la pertinencia del instrumento se observaron promedios por encima del .90 , mientras que en lo que se refiere a la redacción del instrumento los ítems 1, 4 y 13 también reflejan un puntaje mayor a 0.90 como puede verse en la tabla 9 .

Tabla 8. Estadística de fiabilidad

\section{Estadísticas de fiabilidad}

Alfa de Cronbach

Alfa de Cronbach basada en $\mathrm{N}$ de elementos elementos estandarizados

.803

.824

15

Fuente: Elaboración mediante programa estadístico SPSS versión 24.

Tabla 9. Estadística de elemento

\begin{tabular}{|c|c|c|c|}
\hline Media & & ción & $\mathbf{N}$ \\
\hline VAR00001 & 3.8462 & .37553 & 13 \\
\hline VAR00002 & 3.5385 & .77625 & 13 \\
\hline VAR00003 & 3.3846 & .65044 & 13 \\
\hline VAR00004 & 3.6923 & 63043 & 13 \\
\hline VAR00005 & 3.6923 & .63043 & 13 \\
\hline VAR00006 & 3.6923 & .63043 & 13 \\
\hline VAR00007 & 3.5385 & .96742 & 13 \\
\hline VAR00008 & 3.3077 & 1.03155 & 13 \\
\hline VAR00009 & 2.9231 & .75955 & 13 \\
\hline VAR00010 & 3.5385 & .77625 & 13 \\
\hline VAR00011 & 3.9231 & .27735 & 13 \\
\hline VAR00012 & 3.5385 & .77625 & 13 \\
\hline VAR00013 & 3.8462 & .37553 & 13 \\
\hline VAR00014 & 3.6154 & .86972 & 13 \\
\hline VAR00015 & 3.6923 & .63043 & 13 \\
\hline
\end{tabular}

Fuente: Elaboración mediante programa estadístico SPSS versión 24. 


\section{DISCUSIÓN}

El interés por diseñar una rúbrica analítica socioformativa para evaluar la competencia socioemocional de inteligencia emocional, parte de la identificación de la necesidad de contar con instrumentos que, de acuerdo con Barraca, Fernández-González, Opazo y Lozano-Bleda (2012), permitan medir un constructo cuya concepción está en proceso de definición, ya que las investigaciones abordan distinto número de dimensiones, divergencia en las metodologías y diferencias respecto a la interpretación de la calidad de las puntuaciones.

La evaluación de la competencia socio emocional de inteligencia emocional valorando los niveles de desempeño, implica el diseño de una rúbrica donde se describa de forma gradual el impacto que tiene dicha competencia esencial para la conciencia, el desarrollo y la transformación conductual, psicológica y elemental a través de la aplicación y mejoramiento social de y en su contexto, de ahí la relevancia de este estudio, que a través de un juicio de expertos analizado con un tratamiento estadístico con el Alfa de Cronbach, permitió validar con rigor científico un instrumento con enfoque socioformativo, tal como lo plantea Huerta (2016), en el sentido de que la medición de las competencias o habilidades socio emocionales presenta desafíos como son: a) definir cuáles de ellas se deben valorar; b) definir los objetivos de evaluación y seleccionar los instrumentos y métodos de medición; y c) seleccionar medidas que cuenten con propiedades psicométricas sólidas (confiablidad y validez).

La rúbrica analítica socioformativa se postula para explicar regularidades o relaciones que se observan en la conducta y atiende a la validez de la pregunta ¿Hasta dónde el instrumento mide realmente un rasgo determinado y con cuánta eficiencia lo hace? Por lo que sus metas son especificar el significado a evaluar y el significado de sus relaciones con otros instrumentos, así como sus manifestaciones y sus potenciales interpretaciones y aplicaciones en situaciones similares (Prieto y Delgado, 2010).

El análisis de los resultados permitió presentar un seguimiento sistemático y metodológico del diseño de la rúbrica y esto generó una visión integral en el marco de la sociedad del conocimiento como lo argumentan Cardona, Vélez y Tobón (2016). Desde esa perspectiva, tanto la revisión del instrumento por tres expertos, como lo referente a la validez de contenido realizada con el apoyo de veinte jueces expertos, proveen evidencia respecto al grado con el cual los elementos del instrumento de evaluación son 
relevantes y pertinentes (Hayness, Richard y Kubay, 1995; Mendoza y Garza, 2009).

$\mathrm{Si}$ bien otras investigaciones evalúan la inteligencia emocional, por ejemplo con instrumentos como la TESIS de Barraca, Fernández-González, y Sueiro (2009), éste únicamente hace referencia a la capacidad de percibir y comprender las emociones de otros, no las propias; mientras que el MSCEIT (Mayer, Salovey, Caruso y Sitarenios, 2001), adaptado al castellano por Extremera, Fernández-Berrocal y Salovey (2006), evalúa la capacidad de la persona para percibir, responder y manipular la información emocional, así como para comprender y manejar las emociones.

Tobón, Pimienta-Prieto, Herrera-Meza, Juárez-Hernández y Hernández-Mosqueda (2018) argumentan sobre la existencia de vacíos respecto a instrumentos para la medición en diversos ámbitos, tales como las rúbricas con enfoque socioformativo que permiten evaluar las competencias socio emocionales, en ese sentido radicala relevancia de este estudio, en el que a través de un cuestionario diagnóstico se evidencia el proceso de construcción de un instrumento con fundamento y valor científico y que implica la resolución de problemas de contexto para evaluar tres dimensiones: 1) Percepción emocional propia, 2) Percepción emocional de otros y 3) Adaptación y enfoque emocional, con base en una taxonomía socioformativa que los clasifica como: Receptivo, Resolutivo, Autónomo y Estratégico.

Futuras investigaciones abren la posibilidad para aplicar el instrumento diseñado a una muestra representativa, de tal forma que se puedan ofrecer resultados que le permitan al usuario mejorar su inteligencia emocional como una competencia socioemocional con impacto positivo y tangible en su desarrollo personal y profesional.

\section{REFERENCIAS BIBLIOGRAFICAS}

Aiken. L. (1985). Three coefficients for analyzing the reliability and validity of ratings. Educational and Psychological Measurement. 45(1), 131-142 Recuperado de: https://doi.org/10.1177/0013164485451012

Barraca, J., Fernández-González, A., Opazo, H., \& Lozano-Bleda, J. H. (2012). MSCEIT y TESIS: Relaciones entre dos pruebas de habilidad para la evaluación de la Inteligencia Emocional. Recuperado de https://repositorio.ucjc.edu/bitstream/handle/20.500.12020/443/MSCEIT\%20y\% 20TESIS.pdf? sequence $=1$ 
Barraca, J., Fernández-González, A., \& Sueiro, M. (2009). Test de Sensibilidad a las Interacciones Sociales (TESIS): Una prueba objetiva para la medición de la inteligencia emocional. Bizkaia: AlborCohs.

Bisquerra, R. (2003). Educación emocional y competencias básicas para la vida [Emotional education and basic competences for life]. Revista de Investigación $\begin{array}{llll}\text { Educativa } & \text { (RIE), } & \text { 21(1), } & \text { Recuperado de }\end{array}$ https://www.researchgate.net/publication/41570276_Educacion_emocional_y_c ompetencias_basicas_para_la_vida

Cardona, S., Vélez, J., y Tobón, S. (2016). Contribución de la evaluación socioformativa al rendimiento académico en pregrado. Educar, 52(2), 423-447. doi: https://doi.org/10.5565/rev/educar.763

Durlak, J.A., Weissberg, R.P., Dymnicki, A.B., Taylor, R.D., \& Schellinger, K.B. (2011). The Impact of Enhancing Students' Social and Emotional Learning: A MetaAnalysis of School- Based Universal Interventions. Child Development, 82(1), 405-432. Recuperado

de https://www.researchgate.net/publication/49807966_The_Impact_of_Enhancing _Students'_Social_and_Emotional_Learning_A_Meta-Analysis_of_SchoolBased_Universal_Interventions

Extremera, N., Fernández-Berrocal, P., \& Salovey, P. (2006). Spanish version of the Mayer-Salovey-Caruso Emotional Intelligence Test (MSCEIT). Version 2.0: reliabilities, age and gender differences. Psicothema, 18, 42-48.

García-Fernández, M., \& Giménez-Mas, S. I. (2010). Emotional intelligence and its main models: Proposal for an Integrated model. Espiral, 3, 43-52. Recuperado de: http://ojs.ual.es/ojs/index.php/ESPIRAL/article/view/909

Gardner, H. (2011). Frames of mind: The theory of multiple intelligences. UK: Hachette. Haynes, S. N., Richard, D. C. S., \& Kubay, E. S. (1995). Content Validity in Psychological Assessment: A Functional Approach to Concepts and Methods. Psychological Assessment, 7(3), 238-247. Recuperado de: http://citeseerx.ist.psu.edu/viewdoc/download?doi=10.1.1.452.5453\&rep=rep1\& type $=$ pdf 
Hernández-Mosqueda, J.S., Tobón-Tobón, S. \& Guerrero-Rosas, G. (2016). Hacia una evaluación integral del desempeño: las rúbricas socioformativas. Ra Ximhai, 12(6), 359-376.

Huerta, M. (2016). Evaluación de habilidades socioemocionales y transversales: un estado del arte. (s.1.): dialogas, Adelante, Agcid Chile, mesacts y caf-banco de desarrollo de América Latina. Recuperado de: http://www.adelante-i.eu/sites/ default/files/nota_conceptual_taller_evaluacion_caf-final.pdf.

Ibarra-Piza, S., Segredo-Santamaria, S., Juárez-Hernández, L.G. \& Tobón-Tobón, S. ( 2018). Estudio de validez de contenido y confiabilidad de un instrumento para evaluar la metodología socioformativa en el diseño de cursos. Revista Espacios 39 (53).

López-Goñi, I. y Goñi, J.M. (2012). La competencia emocional en los currículos de formación inicial de los docentes. Un estudio comparativo. Revista de Educación, 357 , 467-489. Recuperado de https://dialnet.unirioja.es/servlet/articulo?codigo $=3811857$

Mayer, J. D., Salovey, P., Caruso, D. R., \& Sitarenios, G. (2001). Emotional intelligence as a standard intelligence. Emotion, 1(3).

Mayer, J.D. \& Salovey, P. (1997). What is emotional intelligence? En P. Salovey \& D.J. Sluyter (Eds.). Emotional development and emotional intelligence: Educational implications, (pp. 3-31). New York, USA: Basic Books.

Mendoza, J. G., \& Garza, J. B. (2009). La medición en el proceso de investigación científica: Evaluación de validez de contenido y confiabilidad (Measurement in the scientific research process: Content validity and reliability evaluation). Innovaciones de negocios, 6(11), 17-32.

Montero, I., \& León, O. G. (2005). Sistema de clasificación del método en los informes de investigación en Psicología. International Journal of clinical and health psychology, 5(1), 115-127.

Muñiz, J., Suárez-Álvarez, J., Pedrosa, I., Fonseca-Pedrero, E., \& García-Cueto, E. (2014). Enterprising personality profile in youth: Components and assessment. Psicothema, 545-553

Prieto, G., \& Delgado, A. R. (2010). Fiabilidad y validez. Papeles del Psicólogo, 31, 6774. Recuperado de: http://www.redalyc.org/articulo.oa?id=77812441007 
Tobón, S., Pimienta-Prieto, J. H., Herrera-Meza, S. R., Juarez-Hernandez, L. G., \& Hernandez-Mosqueda, J. S. (2018). Validez y confiabilidad de una rúbrica para evaluar las prácticas pedagógicas en docentes de Educación Media (SOCME-10). Revista Espacios, 39(53).

Welch, S. y Comer, J. 1988). Quantitative Methods for Public Administration: Techniques and Applications. U.S.A.: Editorial Books/Cole Publishing Co.

Zych, I., Beltrán-Catalán, M., Ortega-Ruiz, R., y Llorent, V.J. (2017). Competencias sociales y emocionales de adolescentes involucrados en diferentes roles de bullying y cyberbullying. Revista de Psicodidáctica, 23(2), 86-93. Recuperado de: https://doi.org/10.1016/j.psicod.2017.12.001 